\title{
A COMPARISON OF THE X-RAY SPECTRA OF QUASARS AND BL LAC OBJECTS
}

\author{
D.M. Worrall and B.J. Wilkes \\ Harvard-Smithsonian Center for Astrophysics, Cambridge, MA, U.S.A.
}

\section{INTRODUCTION AND ANALYSIS}

Quasars with similar core-compact radio properties can be classified by their differences at optical and infrared frequencies. Their X-ray properties might be expected to be similar if the synchrotron self-Compton mechanism relates their radio and X-ray emission. We have compared the $0.2-3.5 \mathrm{keV}$ mean power-law energy spectral indices, $\bar{\alpha}$, for 4 quasar classes: 12 Highly Polarized QSOs (HPQs), 19 Flat Radio Spectrum, core-compact, low-polarization, QSOs (FRS QSOs), 24 radio-selected BL Lac objects, and $7 \mathrm{X}$-ray-selected BL Lac objects.

The data were taken from the Einstein Observatory Imaging Proportional Counter (IPC) data bank. We included all the HPQs, FRS QSOs, and BL Lac Objects observed on axis with sufficient counts $(Z 80)$ to allow determination of the IPC gain and spectral fitting. We assumed a powerlaw spectrum with energy index $\alpha$ for each source, and computed the probability distribution of $\alpha$ with normalization and $N_{H}$ (column density of intervening Galactic matter) as free parameters. Multiple observations of the same source were fit simultaneously. The exception was the X-rayselected BL Lac object 2155-304: the only source for which separate observations were inconsistent with a constant spectral index.

Maximum likelihood analysis was used to find the best-fit mean power-law spectral index, $\bar{\alpha}$, and standard deviation, $\sigma$, for each class, assuming the intrinsic distribution is Gaussian.

$$
\text { Model Distribution }=\frac{1}{\sqrt{2 \pi \sigma^{2}}} \exp \left(-\frac{(\alpha-\bar{\alpha})^{2}}{2 \sigma^{2}}\right)
$$

\section{RESULTS}

Results for the HPQs and FRS QSOs are similar, with best-fit mean indices of $\bar{\alpha} \approx 0.5$, whereas for the radio-selected and X-ray-selected BL Lac objects, $\bar{\alpha} \approx 1.0$. Our individual spectral fits for the brightest sources are consistent with those previously published (e.g., Madejski 1985; Wilkes and Elvis 1987). The brightest FRS QSOs fit $N_{H}<$ galactic $N_{H}$ in agreement with Wilkes and Elvis (1987) who infer the presence of soft excess emission. Some sources require $N_{H}>$ galactic $N_{H}$.

Our results are dominated by the brightest sources in each class, but there is no strong evidence that their spectral parameters differ from the weaker sources. For the HPQs, the weakest 10 sources and strongest 2 sources provide approximately equal weights to the results. For the FRS QSOs and radio-selected BL Lac objects the corresponding numbers are 17 and 2, and 18 and 6 , respectively.

The HPQs, FRS QSOs, and radio-selected BL Lac objects are consistent with $\sigma=0$ (see results 
in Figure 1). The Kolmogorov-Smirnov (KS) significance level needed to reject the hypothesis of a constant $\alpha$, and the significance levels when every source is fit to its best-fit $\alpha$ are:

$\begin{array}{rccc} & & \text { Constant } \alpha & \text { Best-Fit } \alpha \\ \text { 19 FRS QSOs } & \alpha=0.5 & 41 \% & 64 \% \\ 12 \text { HPQs } & \alpha=0.5 & 46 \% & 30 \% \\ \text { 24 Radio-selected BL Lac objects } & \alpha=1.0 & 22 \% & 48 \%\end{array}$

In contrast, the X-ray-selected BL Lac objects are inconsistent with a single spectral index. The result is not given entirely by the discrepant measurements of 2155-304.
8 X-ray-selected BL Lac objects
$\alpha=1.1$
$4 \%$
$41 \%$

The KS-test results for fits to each source's best-fit $\alpha$ show that a single-component power-law model is acceptable for the sources in all 4 samples.

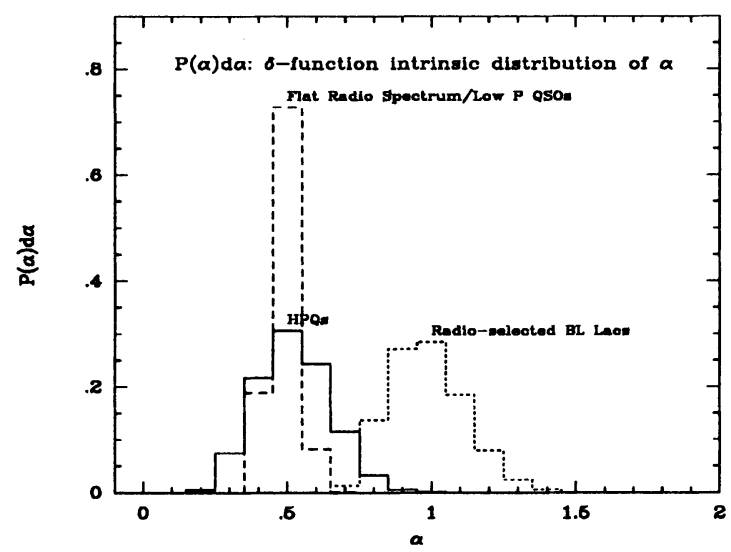

Figure 1. The probability distributions of $\alpha$ for the special case of a constant intrinsic $\alpha$ for each sample $(\sigma=0)$.

\section{DISCUSSION}

BL Lac objects and HPQs display broadly similar continuum spectra from the radio to X-ray wave bands, and they are often treated together in statistical studies under the name 'Blazar'. However, we have found that that their X-ray spectral indices are different on average. This is an intriguing result, but does not necessarily violate a strong connection between the broadband continuum emission of BL Lac objects and QSOs. For example, while superluminal radio components have been detected in members of each class, there is evidence that BL Lac objects are less relativistically beamed at X-ray than radio frequencies (e.g., Maraschi et al. 1986). This may indicate a reduced level of $0.2-3.5 \mathrm{keV}$ Compton radiation in the BL Lac objects with respect to the HPQs and FRS QSOs, and then may explain the difference in X-ray spectral indices.

This work was supported by NASA contract NAS8-30751.

\section{References}

Madejski, G.M. 1985, Ph.D. Thesis, Harvard University.

Maraschi, L., Ghisellini, G., Tanzi, E.G., and Treves, A. 1986, Astrophys. J., 310, 325.

Wilkes, B.J. and Elvis, M. 1987, Astrophys. J., 323, 243. 